\title{
A progenitor candidate for the type II-P supernova SN 2018aoq in NGC 4151
}

\author{
D. O’Neill ${ }^{1}$, R. Kotak ${ }^{2}$, M. Fraser ${ }^{3}$, S. A. Sim ${ }^{1}$, S. Benetti ${ }^{4}$, S. J. Smartt ${ }^{1}$, S. Mattila ${ }^{2}$, C. Ashall ${ }^{5}$, E. Callis ${ }^{3}$, \\ N. Elias-Rosa ${ }^{6,7}$, M. Gromadzki ${ }^{8}$, and S. J. Prentice ${ }^{1}$
}

\author{
1 Astrophysics Research Centre, School of Mathematics and Physics, Queen's University Belfast, Belfast BT7 1NN, UK \\ e-mail: doneil1955@qub.ac.uk \\ 2 Department of Physics and Astronomy, Vesilinnantie 5, University of Turku, 20014 Turku, Finland \\ 3 School of Physics, O’Brien Centre for Science North, University College Dublin, Belfield Dublin 4, Ireland \\ 4 INAF - Osservatorio Astronomico di Padova, Vicolo dell'Osservatorio 5, 35122 Padova, Italy \\ 5 Department of Physics, Florida State University, Tallahassee, FL 32306, USA \\ ${ }^{6}$ Institute of Space Sciences (ICE, CSIC), Campus UAB, Carrer de Can Magrans s/n, 08193 Barcelona, Spain \\ 7 Institut d'Estudis Espacials de Catalunya (IEEC), c/Gran Capitá 2-4, Edif. Nexus 201, 08034 Barcelona, Spain \\ 8 Warsaw University Astronomical Observatory, Al. Ujazdowskie 4, 00-478 Warszawa, Poland
}

Received 2 November 2018 / Accepted 3 December 2018

\begin{abstract}
We present our findings based on pre- and post-explosion data of the type II-Plateau SN 2018aoq that exploded in NGC 4151. As distance estimates to NGC 4151 vary by an order of magnitude, we utilised the well-known correlation between ejecta velocity and plateau brightness, i.e. the standard candle method, to obtain a distance of $18.2 \pm 1.2 \mathrm{Mpc}$, which is in very good agreement with measurements based on geometric methods. The above distance implies a mid-plateau absolute magnitude of $M_{V}^{50}=-15.76 \pm 0.14$ suggesting that it is of intermediate brightness when compared to IIP SNe such as SN 2005cs at the faint end, and more typical events such as SN $1999 \mathrm{em}$. This is further supported by relatively low expansion velocities (Fe II $\lambda 5169 \sim 3000 \mathrm{~km} \mathrm{~s}^{-1}$ at $+42 \mathrm{~d}$ ). Using archival HST/WFC3 imaging data, we find a point source coincident with the supernova position in the F350LP, F555W, F814W, and F160W filters. This source shows no significant variability over the $\sim 2$ month time span of the data. From fits to the spectral energy distribution of the candidate progenitor, we find $\log \left(L / L_{\odot}\right) \sim 4.7$ and $T_{\text {eff }} \sim 3.5 \mathrm{kK}$, implying an M-type red supergiant progenitor. From comparisons to single and binary star models, we find that both favour the explosion of a star with a zero-age main sequence mass of $\sim 10 M_{\odot}$.
\end{abstract}

Key words. stars: evolution - supernovae: individual: SN 2018aoq - supernovae: general - galaxies: individual: NGC 4151

\section{Introduction}

Although type II-plateau $(\mathrm{P})$ supernovae $(\mathrm{SNe})$ are the most commonly occurring subtype of explosions resulting from the core-collapse (CC) of massive evolved stars (Li et al. 2011), a number of issues remain to be explored relating to their evolution prior to collapse and the origin of diversity in their observed properties. Direct detections of sources at the SN site in preexplosion imaging have led to a growing body of evidence in favour of red supergiants (RSG) being the progenitors of type IIP SNe (van Dyk et al. 2003; Smartt et al. 2004). Based on the consideration of a sample of 20 IIP SNe within $25 \mathrm{Mpc}$ and a combination of detections and limits to the presence of a point source at the SN location, the progenitors have inferred masses in the range $8.5_{-1.5}^{+1.0} \lesssim M_{\odot} \lesssim 16.5_{-1.5}^{+1.5}$ (Smartt et al. 2009).

There is considerable diversity in the observed photometric and spectroscopic behaviour of type IIP SNe. At the faint end of the IIP brightness distribution $\left(-13 \lesssim M_{V} \lesssim-15\right)$, it is arguably natural to assume that they might simply originate from stars that are only just massive enough to undergo Fe CC. Indeed, there is some evidence to support this view from progenitor detections, for example SNe 2005cs and 2008bk (Maund et al. 2005, 2014; Mattila et al. 2008; van Dyk et al. 2012). However, it is plausible that faint $\mathrm{SNe}$ arising from $\mathrm{CC}$ due to electron captures on ${ }^{24} \mathrm{Mg}$ and ${ }^{20} \mathrm{Ne}$ in the cores of super asymptotic giant branch stars (Nomoto 1984) also play a role. Uncertainties in modelling processes such as mass loss, mixing, and convection together with the influence of metallicity have resulted in a wide mass range $\left(7-11 M_{\odot}\right)$ over which electron-capture $\mathrm{SNe}$ can occur. Although the picture is likely to be far more complicated than described above, gathering progenitor masses estimated from multi-band pre-explosion imaging, provides valuable information against which models can be anchored.

SN 2018aoq (Kait-18P) was discovered on 2018 April 01.436 by the Lick Observatory Supernova Search (LOSS) at +15.3 mag unfiltered magnitude (Nazarov et al. 2018) on the edge of the intermediate spiral Seyfert galaxy NGC 4151 (Fig. A.1). It was spectroscopically classified as a type II SN on 2018 April 02 (Yamanaka 2018). Based on early indications that it might be fainter than the typical type IIP population, and motivated by the abundance of pre-explosion imaging of NGC 4151, we initiated a follow-up campaign as described below. 


\section{Observations and data reduction}

Optical imaging data were collected primarily using a combination of ATLAS (Asteroid Terrestrial-impact Last Alert System; Tonry et al. 2018) and the $2.0 \mathrm{~m}$ Liverpool Telescope (LT). Spectroscopic follow-up was carried out primarily at the $2.5 \mathrm{~m}$ Nordic Optical Telescope (NOT) as part of the NOT Unbiased Transient Survey $\left(\right.$ NUTS $\left.^{1}\right)$. A complete record of photometric and spectroscopic data can be found in Tables A.1, A.2, and A.4. Standard procedures of bias subtraction, trimming the overscan regions, and flat-field correction were applied to the raw imaging and spectroscopic data. Instrumental magnitudes for the SN photometry were found by fitting a point spread function (PSF) with the SNOoPy IRAF package ${ }^{2}$ to the reference stars listed in Table A.5 and subtracting this PSF from the SN. The photometric zero point corrections for both the LT and NOT images were calculated using the instrumental magnitudes of the stars in the field (Table A.5) and comparing these to the magnitude values given by SSDS DR12 (Alam et al. 2015) in each of the filters. In the case of the $B$ and $V$ bands, the magnitudes in the Sloan bands were transformed using the transformations listed in Jester et al. (2005). For the spectroscopic data, the wavelength calibration was carried out using $\mathrm{He}-\mathrm{Ne}$ arcs, and relative flux calibration was performed using spectrophotometric standards taken on the same night, and with the same instrumental configuration as for SN 2018aoq. Absolute flux calibration of the spectra was achieved using the broadband imaging points interpolated to the epochs of spectroscopy. From the ATLAS nondetection on 2018 March 28 and the discovery on 2018 April 01 (Fig. 1, Table A.2), we adopt 2018 March $30(\mathrm{MJD}=58208)$ as the explosion date.

\section{Reddening and distance}

The Galactic reddening in the line of sight to SN 2018aoq is found to be $E(B-V)=0.02$ (Schlafly \& Finkbeiner 2011). We attempt to estimate the host galaxy reddening by comparing the colour evolution and spectral shape to other type IIP SNe. Figure 1 shows the $V-I$ and $R-I$ colour evolution of SN 2018aoq compared to a sample of well-observed IIP SNe (Table A.6). A fairly uniform colour evolution is expected over the first $\sim 100 \mathrm{~d}$ as hydrogen recombination dominates (Eastman et al. 1996). A least squares fit using low-order polynomials was carried out on the extinction-corrected colour curves of the sample SNe. The $V-I$ and $R-I$ points of SN 2018aoq were then reddened by iteratively varying the $E(B-V)$ value until the best fit was obtained. The colour evolution of SN 2018aoq tracks the general IIP evolution; a best fit is obtained for the total host and Galactic extinction $E(B-V)_{\text {total }} \sim 0.04$. The $V-I$ colour appears to be systematically bluer than the sample, but still within the range exhibited by other IIP SNe.

Spectra of SN 2018aoq were also compared to the extinctioncorrected spectra of $\mathrm{SNe} 1999 \mathrm{em}$ and $2005 \mathrm{cs}$ (with $\mathrm{E}(\mathrm{B}-$ $\mathrm{V})$ values of 0.1 and $0.05 \mathrm{mag}$, respectively; Elmhamdi et al. 2003; Pastorello et al. 2009) at similar epochs. We reddened the SN 2018aoq spectra incrementally until a reasonable match was obtained; we found this value to be $E(B-V) \sim 0.03$. Also, we do not clearly detect any feature attributable to the $\mathrm{Na} I \mathrm{D}$ lines in our earliest spectrum.

We further note that the location of the $\mathrm{SN}$ in a relatively isolated region at $\sim 73^{\prime \prime}$ from the nucleus of the host galaxy is in line with the low inferred extinction. Based on the findings

\footnotetext{
1 http://csp2.1co.cl/not/

2 http://sngroup.oapd.inaf.it/snoopy.html
}

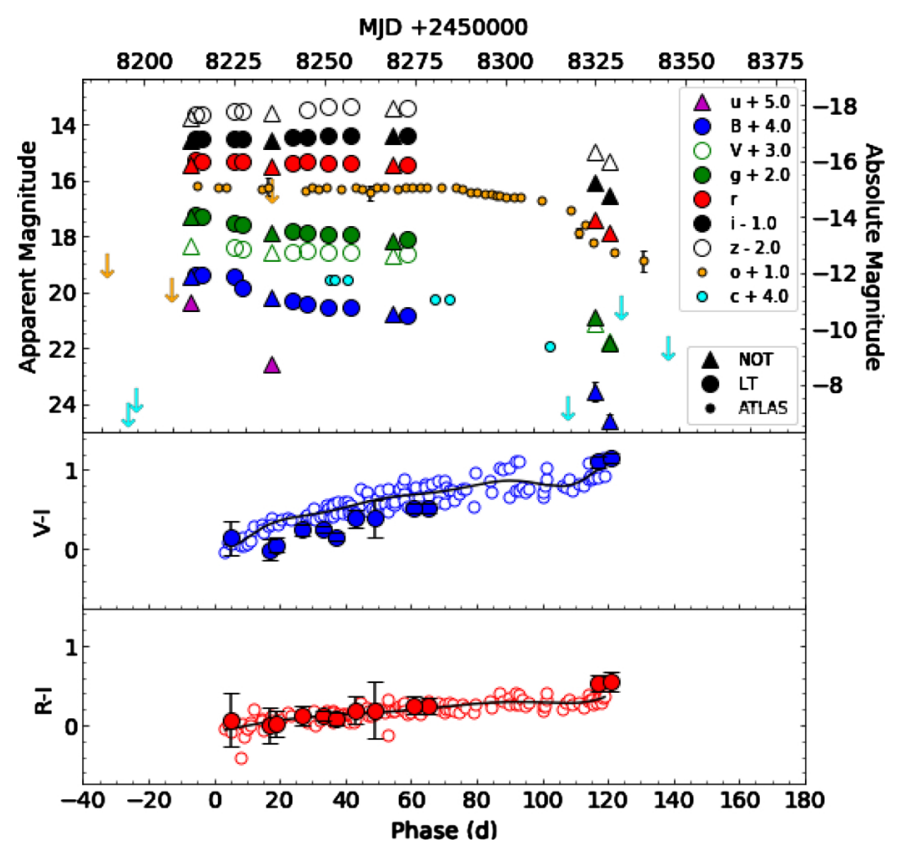

Fig. 1. Top panel: light curves of SN 2018aoq in various filters. Upper limits are indicated by downward pointing arrows. The letters "c" and "o" indicate the "cyan" ( $\left.\lambda_{\mathrm{eff}} \sim 5330 \AA\right)$ and "orange" $\left(\lambda_{\mathrm{eff}} \sim 6790 \AA\right)$ ATLAS filters, respectively. The non-detection (>18.85 mag) on 2018 March $28(\mathrm{MJD}=58206)$ allows us to constrain the rise time to $\lesssim 5 \mathrm{~d}$. Bottom panel: extinction-corrected $V-I$ and $R-I$ colour evolution for SN 2018aoq. The errors are the result of compounding errors in transforming from the $V, r$, and $i$ filters to Johnson $R$ and $I$. Open circles are extinction-corrected values taken from 8 well-sampled type IIP SNe (Table A.6). The black line indicates a fit to these data from which we infer $E(B-V)=0.04$ for SN 2018aoq.

above, we conclude that the SN suffers from low extinction and adopt of a value of $E(B-V)_{\text {tot }}=0.04$ mag in what follows.

There is a great variation in the estimated distances to NGC 4151, ranging from $3.8-29.2 \mathrm{Mpc}$ as listed on $\mathrm{NED}^{3}$. NGC 4151 has received considerable attention over the years as it is one of the very few local AGN for which dynamical mass estimates of the central black hole can be compared to those obtained from reverberation mapping and other methods, thereby providing a calibration of the black hole mass scale. However, anchoring the calibration to NGC 4151 requires a reliable distance to it. Using the heliocentric radial velocity of the galaxy is $998 \mathrm{~km} \mathrm{~s}^{-1}$ (Pedlar et al. 1992) and yields a distance of $14.1 \mathrm{Mpc}$ with $H_{0}=71 \mathrm{~km} \mathrm{~s}^{-1} \mathrm{Mpc}^{-1}$. However, strong peculiar motions render this value unreliable. Distance estimates using the Tully-Fisher method do not fare much better as the orientation of NGC 4151 is almost perpendicular to our line of sight, making measurements of the rotational velocity challenging. Based on a variant of the quasar parallax method proposed by Elvis \& Karovska (2002) to obtain direct distances to quasars, Hönig et al. (2014) reported a value of $19_{-2.6}^{+2.4} \mathrm{Mpc}$. This value is reliable as it depends on purely geometrical arguments rather than requiring an underlying source of known luminosity.

In order to obtain an independent estimate for the distance, we applied the standard candle method (SCM) as described in Hamuy \& Pinto (2002). Essentially, the SCM is the manifestation of a positive correlation between the luminosity and expansion velocity during the plateau phase; a convenient reference point is taken to be at mid-plateau ( $\sim 50 \mathrm{~d})$. Fortuitously, we have

\footnotetext{
https://ned.ipac.caltech.edu/
} 
$V$ and $i$-band measurements taken on May $18,49 \mathrm{~d}$ post explosion (Table A.1); to convert to the $I$-band, we used the transformations in Jester et al. (2005) to find a value of $I=15.25 \pm 0.29$ mag. The Fe II $\lambda 5169$ velocity at $50 \mathrm{~d}$ was found by linear interpolation between the velocity values found at $+42 \mathrm{~d}$ and $+61 \mathrm{~d}$, which yielded a value of $2699 \pm 120 \mathrm{~km} \mathrm{~s}^{-1}$. We used the sample provided by Polshaw et al. (2015) consisting of type IIP SNe that occurred in galaxies with Cepheid distances for calibration.

We find $D_{I}=18.9 \pm 1.5 \mathrm{Mpc}$ and $D_{V}=17.3 \pm 1.8 \mathrm{Mpc}$ with a weighted average of $18.2 \pm 1.2 \mathrm{Mpc}$, which is the value we adopt in this work. We note that this value is in excellent agreement with the value based on direct methods (Hönig et al. 2014).

\section{Supernova photometry and spectroscopy}

Using the values for the distance and reddening from Sect. 3, we find $M_{V}=-15.76 \pm 0.14$ at an epoch of $49 \mathrm{~d}$, i.e. approximately mid-plateau. Thus, SN 2018aoq appears to lie at a brightness intermediate between the normal and subluminous IIP populations, although the demarcations in brightness between these groups are not strictly defined. We note in passing that the rise time to the plateau was relatively rapid ( $\lesssim 5 \mathrm{~d}$; Fig. 1 ), in keeping with previous studies, for example Gal-Yam et al. (2011) and Gall et al. (2015), who reported a possible correlation between rise time and absolute peak brightness.

From a blackbody fit to the photometric data at $+49 \mathrm{~d}$, we estimate a quasi-bolometric luminosity of $L_{\mathrm{Bol}}=$ $10^{41.8} \mathrm{erg} \mathrm{s}^{-1}$. Not surprisingly, this is fainter than that of SN 1999em, a normal II-P and marginally brighter than SN $2005 \mathrm{cs}, L_{\mathrm{Bol}}=10^{41.4-41.7} \mathrm{erg} \mathrm{s}^{-1}$ (Bersten \& Hamuy 2009; Pastorello et al. 2009). The photometric data (Tables A.1, A.2) allow us to constrain the plateau duration to $\sim 80 \mathrm{~d}$ following which the light curve drops at a rate of $0.02 \mathrm{mag} \mathrm{day}^{-1}$ ( $o$-band) for $\sim 20 \mathrm{~d}$, when it abruptly drops onto the nebular tail phase. This drop is $\sim 2$ mag compared to the mid-plateau brightness and is shallower than that of SN $2005 \mathrm{cs}$ in a comparable filter $\left(m_{R}=2.7 \mathrm{mag}\right.$; Pastorello et al. 2009).

The 4-111 d spectral evolution of SN 2018aoq is shown in Fig. A.4. The spectra are typical of type IIP SNe; the early spectra are blue, which supports our finding (cf. Sect. 3) of minimal extinction. The Balmer lines and He I $\lambda 5875$ are clearly discernible in the spectra. By $42 \mathrm{~d}$, the metal lines for instance Fe II $\lambda 5169, \mathrm{Ba}$ II $\lambda 6142$, and Sc II $\lambda 6246$ have all emerged, as has the Ca II triplet. By $61 \mathrm{~d}$, the forbidden [Ca II] 17291 emerges - all of these features are expected to be present in type IIP $\mathrm{SN}$ spectra at these epochs. From the absorption minimum in the $4 \mathrm{~d}$ spectrum, we measure an $\mathrm{H} \alpha$ velocity of $\sim 9700 \mathrm{~km} \mathrm{~s}^{-1}$, which is $\sim 2600 \mathrm{~km} \mathrm{~s}^{-1}$ slower than the corresponding velocity measured for the earliest spectra available for SN $1999 \mathrm{em}$ (+5d, Leonard 2002). Likewise, the velocities measured from the metal lines in the $42 \mathrm{~d}$ spectrum are $\sim 800 \mathrm{~km} \mathrm{~s}^{-1}$ lower than those of typical IIP SNe such as SN 1999em (Elmhamdi et al. 2003 ) at similar epochs, but $\sim 800 \mathrm{~km} \mathrm{~s}^{-1}$ higher than those from the low-luminosity group such as SN2005cs (Pastorello et al. 2009): $2960 \pm 60 \mathrm{~km} \mathrm{~s}^{-1}$ and $2689 \pm 60 \mathrm{~km} \mathrm{~s}^{-1}$, measured for Fe II $\lambda 5196$ and Sc II $\lambda 6246$, respectively. A spectral comparison with SNe $1999 \mathrm{em}$ and $2005 \mathrm{cs}$ that illustrates the above is shown in Fig. A.2.

\section{Archival data and progenitor identification}

A wealth of pre-explosion archival data are available for NGC 4151. We focus primarily on pre-explosion WFC3 imaging data from the Hubble Space Telescope (HST) taken
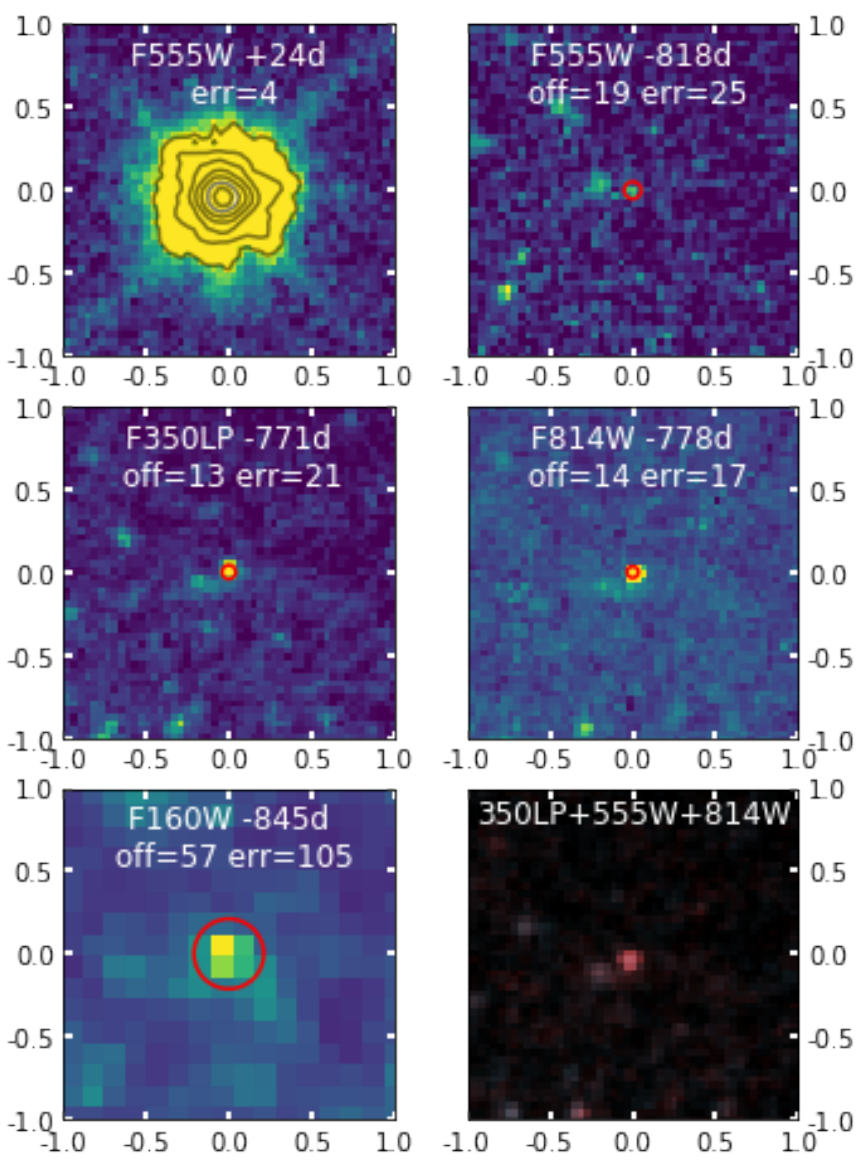

Fig. 2. Top left panel: $2^{\prime \prime} \times 2^{\prime \prime}$ HST/WFC3 image stamps. It shows SN 2018aoq at an epoch of $24 \mathrm{~d}$ after explosion while all others show pre-explosion images (Table A.3). The filter and epoch are labelled. The offset between the star and SN position, and the associated positional error (in mas) is also indicated. The centroid of the SN position, as measured from the image in the top left panel, is shown with a red circle, the radius of which is twice the uncertainty in the position for clarity. The limiting and absolute magnitudes for the progenitor in each filter are $m_{F 555 W}>28.0(-3.3), m_{F 350 L P}>26.8(-4.5), m_{F 814 W}>26.0(-5.3)$, and $m_{F 160 W}>24.1(-7.2)$.

approximately $2 \mathrm{yr}$ prior to the occurrence of SN 2018aoq (Table A.3). Luckily, a post-explosion image $(+24 d)$ in the F555W filter also taken with the WFC3 instrument was publicly available ${ }^{4}$, allowing us to accurately map the position of the SN to pre-explosion images. The position of the SN in the pre-explosion drizzled images was found using a linear transformation computed within the geomap and geoxytran tasks in IRAF using five to six field stars in common. Although the field of view of the WFC3 instrument is $162^{\prime \prime} \times 162^{\prime \prime}$, the difference in exposure times of the pre- and post-explosion F555W images meant that only a few stars were available in the two F555W images to calculate the coordinate transformation. However, as previously noted, SN 2018aoq does not lie in a crowded field.

Figure 2 shows the result of the above procedure in various filters. The $3 \sigma$ detection limit in each filter was found by adding artificial stars created using TinyTim ${ }^{5}$. The artificial stars were then scaled such that the peak count of the PSF matched the background noise $+3 \sigma$. Photometry on these artificial stars was then carried out as previously described. The limits are given in

\footnotetext{
HST Proposal ID:15151.

http://www. stsci.edu/hst/observatory/focus/TinyTim
} 
Fig. 2. A bright point source was identified in the images very close to the SN position. For the F555W pre- and post-explosion images, the offset between the SN centroid and the point source was found to be 19 mas.

In order to robustly estimate the errors, we ran Monte Carlo simulations on synthetic data. The position of the peak was allowed to vary from -100 mas to +100 mas in both directions in steps of 0.4 mas. The process was repeated for 1000 iterations. The discrepancy between this position and that found previously is taken to be representative of the error. The total error in position is the combination, in quadrature, of the positional errors in the $\mathrm{SN}$ and pre-explosion source, as well as the error in the transformation. For the F555W filter (available for both pre- and post-explosion epochs), we find the error to be 25 mas. For all filters, we find the offsets to be within the total error estimate (Fig. 2), and therefore conclude that the source is coincident with SN 2018aoq.

Photometry on the star was carried out using DOLPHOT ${ }^{6}$. The measurements for the star in all available filters are listed in Table A.3 and shown in Fig. A.3; The progenitor star had a mean absolute magnitude in the optical bands of $M_{F 350 L P}=-5.69$, $M_{F 555 W}=-4.74, M_{F 814 W}=-7.39$ and in the near-infrared: $M_{F 160 W}=-9.48$. The pre-explosion photometry covers a period of $73 \mathrm{~d}$ over the course of which there appears to be little or no variation within a few tenths of a magnitude in any of the filters.

In order to characterise its properties, we used the HST synthetic photometry package stsynphot ${ }^{7}$. From the measured average apparent magnitude in each filter, we generated a spectral energy distribution (SED) using models taken from the PHOENIX stellar atlas (Husser et al. 2013). Using the prescriptions provided by Kochanek et al. (2012) for circumstellar dust, we simultaneously varied the temperature, extinction, $\log$ surface gravity $(\mathrm{g})$, and optical depth $\left(\tau_{V}\right)$ of the circumstellar dust to generate synthetic magnitudes in the HST filters and compared these with our measured values. The best-fitting parameters, i.e. those that returned the smallest mean scatter ( $\sigma=0.056 \mathrm{mag}$ ), were found to be a temperature of $T=$ $3500 \pm 150 \mathrm{~K}, \log (g)=0.0$ and minimal circumstellar extinction $\tau_{V}=0.0$. The resulting SED is shown in Fig. 3 .

For completeness, we note that no source is visible in preexplosion imaging at 3.6 and $4.8 \mu \mathrm{m}$ in Spitzer/IRAC imaging down to limiting Vega magnitudes of $>20.7$ and $>20.1$ (from artificial star tests), respectively; these are consistent with the SED.

Integrating over the SED allows us to obtain the total flux from the progenitor, which can then be used to constrain the luminosity and radius. In order to explore a conservative parameter range, we folded into the luminosity bounds the entire range of SCM distances implied by the uncertainties (i.e. $15.5-20.4 \mathrm{Mpc}$ ) in Sect. 3. We found a luminosity range of $\log \left(L / L_{\odot}\right)=4.59-4.83(4.72 \pm 0.12)$ for the progenitor candidate, making it a likely red supergiant of spectral type $M$. To estimate its initial mass, we considered evolutionary tracks computed assuming solar metallicity for both single- and binarystar models that contain similar treatments of the dominant physical processes (STARS, BPASSv2.1, Eggleton et al. 2011; Eldridge et al. 2017, respectively). Models were selected by comparing the inferred luminosity and temperature to the calculated values.

\footnotetext{
6 http://americano.dolphinsim.com/dolphot/

https://stsynphot.readthedocs.io/en/latest/
}

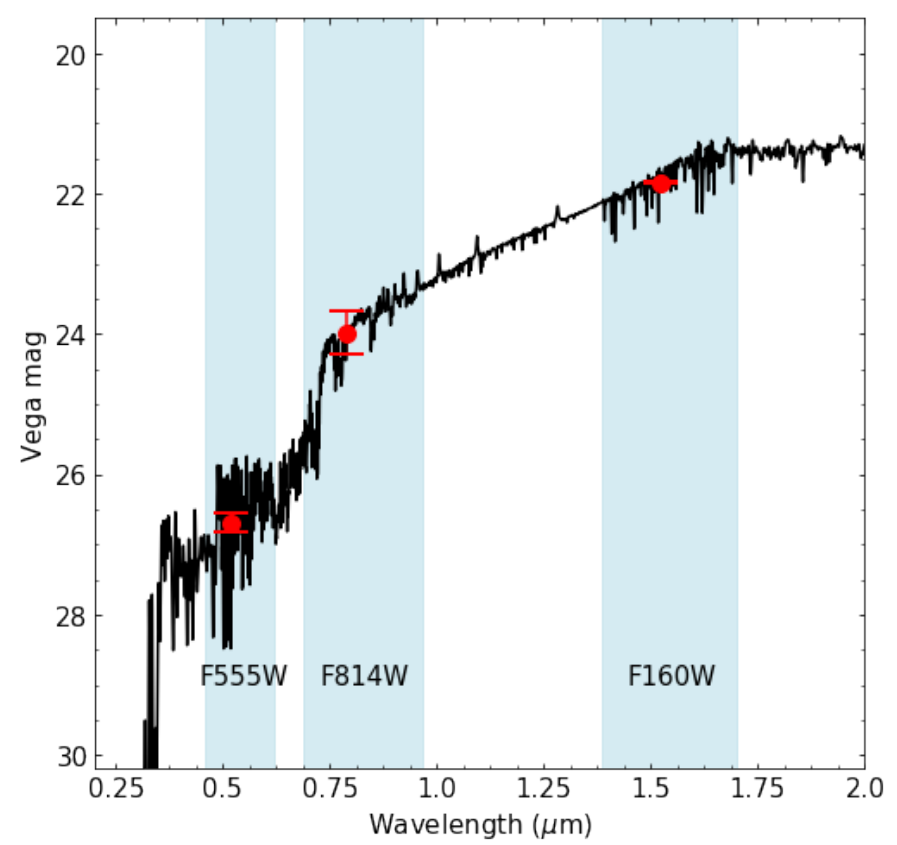

Fig. 3. Best-fitting synthetic SED from the PHOENIX stellar atmosphere models corresponding to spectral-type $\mathrm{M}$ with $T=3500 \mathrm{~K}$, $\log g=0.0$ and $E(B-V)=0.03$, in units of Vega mag. The average scatter between the SED and observed HST photometry $\sigma=0.05$ mag. The shaded regions indicate the wavelength range of each of the filters. The red points denote the average measured magnitude in each of the HST filters.

For the single star case, three models matched the progenitor properties with masses in the $9-11 M_{\odot}$ range (Fig. 4 left panel). A comparison with the non-rotating Geneva models (Meynet et al. 1994), based on a slightly coarser mass grid, is consistent with the above, yielding a likely progenitor in the range $8 \leq M_{\odot} \leq 12$. We found 253 binary models that matched the progenitor properties out of $\approx 12600$ BPASS models considered. The vast majority $(\sim 80 \%)$ of these consisted of a 9-11 $M_{\odot}$ star in a long period orbit $(P \gtrsim 1000$ days) with a range of companion masses $\left(1 M_{\odot}-10 M_{\odot}\right)$. The wide orbits mean minimal mass transfer between the primary and secondary stars during the course of their respective evolutionary phases. Thus, the mass of the companion is of little consequence. The remaining models result in mergers between two lower mass stars in tight orbits with $P \lesssim 10$ days, resulting in a star with a mass close to $10-11 M_{\odot}$. Thus, both the single- and binary star models favour a star in the range $10 \pm 2 M_{\odot}$. There is still some uncertainty regarding the temperature range of RSG stars and the methods used to derive them (Davies et al. 2013; Levesque et al. 2006). In order to ensure that our results are not highly dependent on the estimated temperature, we increased the temperature range considered to $2500-4500 \mathrm{~K}$ and repeated the process. We found the results to be entirely consistent with the above.

From the HST photometry and SED (Fig. 3), we can rule out a bright companion star. A single SED matches the HST photometry very well, so a companion would have to have a SED that either peaks sharply in the ultraviolet region or in the midinfrared. The latter is unlikely, given the non-detections in the Spitzer/IRAC bands. In order to determine the robustness of the above statements, we combined the progenitor SED with that obtained from synthetic spectra with $2000 \lesssim T_{\text {eff }} \lesssim 30000$ and $10 \lesssim$ $m_{F 814 W} \lesssim 30$ to examine whether it might possible for a companion to exist alongside the progenitor, but without affecting the 


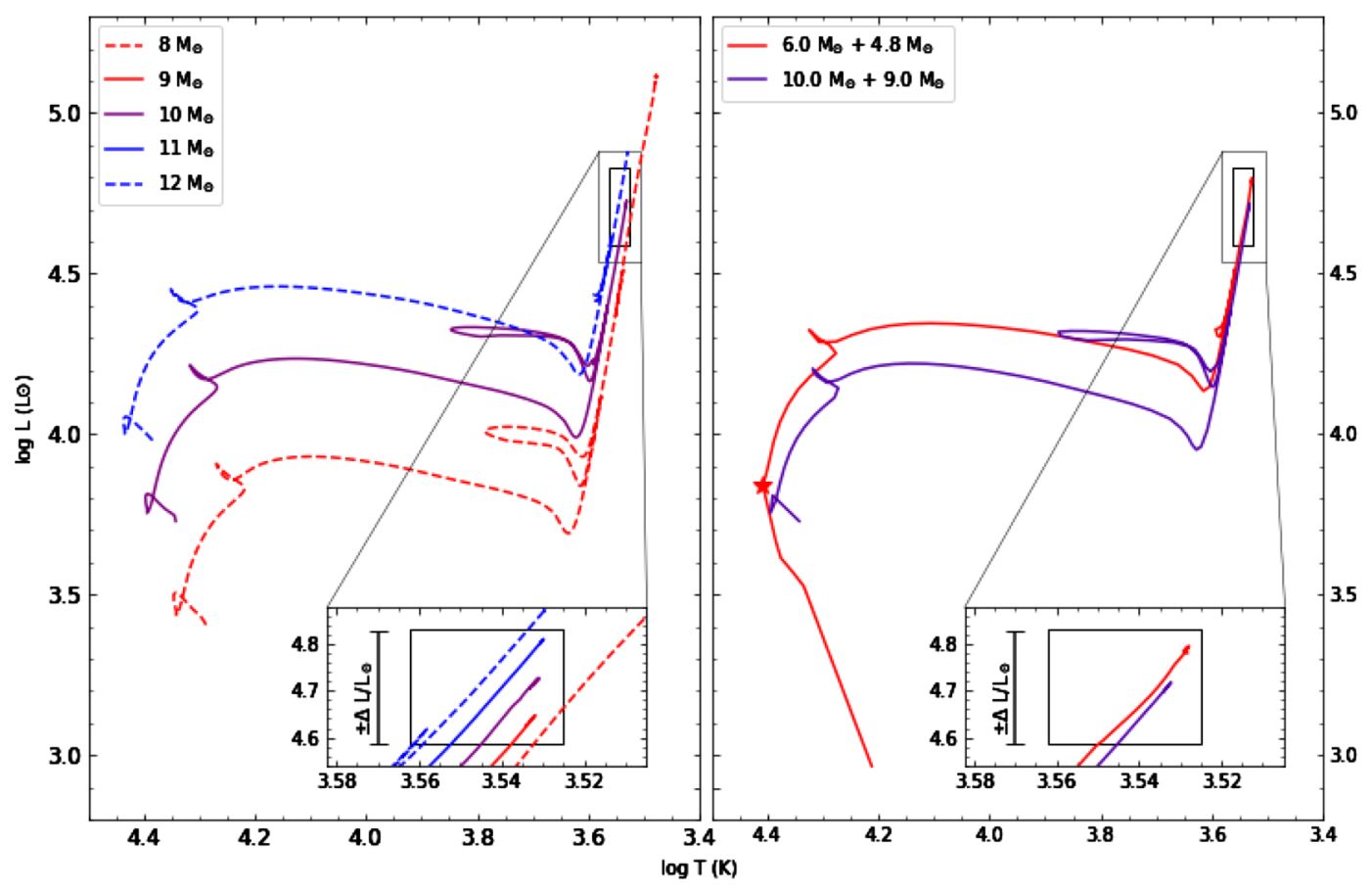

Fig. 4. Evolutionary tracks computed at solar metallicity $(Z=0.02)$ and selected to satisfy the inferred temperature and luminosity of progenitor as described in Sect. 5 and delineated by the box. Left panel: single star models from the STARS code. 9-11 $M_{\odot}$ models matched the progenitor properties at its endpoint. The $8 M_{\odot}$ and $12 M_{\odot}$ tracks are shown for comparison. The inset shows a zoom-in of a finer grid of models with masses as indicated in the legend. Right panel: evolutionary tracks resulting from binary evolution computed using the BPASS code. In total, 253 tracks matched the progenitor properties. These had primary stars in the mass range $6-10 M_{\odot}$, and $50 \%$ had a $10 M_{\odot}$ primary. Shown here is a $10 M_{\odot}$ primary with a $9 M_{\odot}$ secondary on a long period orbit ( 4000 d) with little interaction (blue) and an early merger between stars in a small orbit( $\sim 10 \mathrm{~d})$, which initially consisted of a $6 M_{\odot}$ primary and $4.8 M_{\odot}$ secondary resulting in a star with a mass between $10 M_{\odot}$ and $11 M_{\odot}($ red). The red star indicates the position on the isochrone at which the merger was completed.

SED (Fig. 3). This exercise also allows us to assess the feasibility of detecting a companion once the SN has faded away. We were unable to find such a case within the above range of parameters as any companion star would be fainter than $M_{F 814 W}=-1.3$ ).

Therefore, we conclude that the progenitor was a single star with $M_{\text {ZAMS }}=10 \pm 2 M_{\odot}$, in a wide binary with a low mass companion, or that it resulted from the merger of two low mass stars with a combined mass close to $M_{\text {ZAMS }}=10 \pm 2 M_{\odot}$. In either of the above cases, a clear prediction would be that very late time imaging observations should show that the source we identified as the progenitor has disappeared because a putative companion would be too faint to detect. Similar results based on an in-depth study of model dependencies related to type II SNe has been found by Zapartas (2018).

\section{Summary}

We have presented the case for the detection of the progenitor to the II-P SN 2018aoq. Using pre-explosion data from HST, we find that the SED best matches a RSG star that has a temperature of $\sim 3500 \pm 150 \mathrm{~K}$ and luminosity of $\log \left(L / L_{\odot}\right) \sim 4.7$, has minimal Galactic and circumstellar dust extinction, and displays minimal variability in the lead up to CC. Both single and binary star models favour a progenitor with a ZAMS mass of $10 \pm 2 M_{\odot}$. The minimal extinction and isolated location of SN 2018aoq lends confidence to the progenitor detection, making it one of only a few for which multi-filter, multi-epoch observations were possible. Although further observations will be required to constrain the ${ }^{56} \mathrm{Ni}$ mass and to confirm the disappearance of the progenitor, our result adds to the small but growing body of evidence in favour of the explosion of low mass RSGs leading to faint type IIP SNe. Although previous studies have explored the possibility of a link between progenitor mass and explosion energy (e.g. Poznanski 2013), from an observational standpoint, the existence (or otherwise) of such a correlation will have to await a larger sample of robust progenitor detections.

Acknowledgements. We acknowledge comments from an anonymous referee. We thank JJ Eldridge for patiently answering questions related to BPASS. D. O'Neill acknowledges a DEL studentship award. MF acknowledges the support of a Royal Society - Science Foundation Ireland University Research Fellowship. This work is based on observations made with the Nordic Optical Telescope, operated by the Nordic Optical Telescope Scientific Association at the Observatorio del Roque de los Muchachos, La Palma, Spain, of the Instituto de Astrofisica de Canarias. The Liverpool Telescope is operated on the island of La Palma by Liverpool John Moores University in the Spanish Observatorio del Roque de los Muchachos of the Instituto de Astrofisica de Canarias with financial support from the UK Science and Technology Facilities Council. The observations were made as part of the JL18A10b programme. Some of the data presented in this paper were obtained from the Mikulski Archive for Space Telescopes (MAST). STScI is operated by the Association of Universities for Research in Astronomy, Inc., under NASA contract NAS5-26555. This work is also based on data from HST proposal: 15151 (S. V. Dyk) retrieved from the MAST archive. This work has made use of data from the Asteroid Terrestrialimpact Last Alert System (ATLAS) project. ATLAS is primarily funded to search for near-Earth asteroids through NASA grants NN12AR55G, 80NSSC18K0284, and $80 \mathrm{NSSC} 18 \mathrm{~K} 1575$; byproducts of the NEO search include images and catalogues from the survey area. The ATLAS science products have been made possible through the contributions of the University of Hawaii Institute for Astronomy, the Queen's University Belfast, and the Space Telescope Science Institute. SJS acknowledges funding from STFC Grants ST/P000312/1 and ST/N002520/1. N.E-R. acknowledges support from the Spanish MICINN grant ESP2017-82674$\mathrm{R}$ and FEDER funds. MG is supported by the Polish National Science Centre grant OPUS 2015/17/B/ST9/03167. 


\section{References}

Alam, S., Albareti, F. D., Allende Prieto, C., et al. 2015, ApJS, 219, 12 Baron, E., Branch, D., \& Hauschildt, P. H. 2007, ApJ, 662, 1148 Bersten, M. C., \& Hamuy, M. 2009, ApJ, 701, 200

Davies, B., Kudritzki, R.-P., Plez, B., et al. 2013, ApJ, 767, 3

Eastman, R. G., Schmidt, B. P., \& Kirshner, R. 1996, ApJ, 466, 911 Eggleton, P. P., Tout, C., Pols, O., et al. 2011, Astrophysics Source Code Library, [record ascl:1107.008]

Eldridge, J. J., Stanway, E. R., Xiao, L., et al. 2017, PASA, 34, e058

Elmhamdi, A., Danziger, I. J., Chugai, N., et al. 2003, MNRAS, 338, 939

Elvis, M., \& Karovska, M. 2002, ApJ, 581, L67

Fraser, M., Ergon, M., Eldridge, J. J., et al. 2011, MNRAS, 417, 1417

Gal-Yam, A., Kasliwal, M. M., Arcavi, I., et al. 2011, ApJ, 736, 159

Gall, E. E. E., Polshaw, J., Kotak, R., et al. 2015, A\&A, 582, A3

Hamuy, M., \& Pinto, P. A. 2002, ApJ, 566, L63

Hamuy, M., Pinto, P. A., Maza, J., et al. 2001, ApJ, 558, 615

Hendry, M. A., Smartt, S. J., Maund, J. R., et al. 2005, MNRAS, 359, 906

Hönig, S. F., Watson, D., Kishimoto, M., \& Hjorth, J. 2014, Nature, 515, 528

Husser, T.-O., Wende-von Berg, S., Dreizler, S., et al. 2013, A\&A, 553, A6

Jester, S., Schneider, D. P., Richards, G. T., et al. 2005, AJ, 130, 873

Kochanek, C. S., Khan, R., \& Dai, X. 2012, ApJ, 759, 20

Leonard, D. C. 2002, PASP, 114, 1291

Levesque, E. M., Massey, P., Olsen, K. A. G., et al. 2006, ApJ, 645, 1102

Li, W., Leaman, J., Chornock, R., et al. 2011, MNRAS, 412, 1441

Mattila, S., Smartt, S. J., Eldridge, J. J., et al. 2008, ApJ, 688, L91
Maund, J. R., Smartt, S. J., \& Danziger, I. J. 2005, MNRAS, 364, L33

Maund, J. R., Mattila, S., Ramirez-Ruiz, E., \& Eldridge, J. J. 2014, MNRAS, 438, 1577

Meynet, G., Maeder, A., Schaller, G., Schaerer, D., \& Charbonnel, C. 1994, A\&AS, 103, 97

Nazarov, S. V., Okhmat, D. N., Sokolovsky, K. V., \& Denisenko, D. V. 2018, ATel, 11498

Nomoto, K. 1984, ApJ, 277, 791

Pastorello, A., Zampieri, L., Turatto, M., et al. 2004, MNRAS, 347, 74

Pastorello, A., Valenti, S., Zampieri, L., et al. 2009, MNRAS, 394, 2266

Pedlar, A., Kukula, M., Longley, D., et al. 1992, in Physics of Active Galactic Nuclei, eds. W. J. Duschl, \& S. J. Wagner, 338

Polshaw, J., Kotak, R., Chambers, K. C., et al. 2015, A\&A, 580, L15

Poznanski, D. 2013, MNRAS, 436, 3224

Roy, R., Kumar, B., Benetti, S., et al. 2011, ApJ, 736, 76

Schlafly, E. F., \& Finkbeiner, D. P. 2011, ApJ, 737, 103

Smartt, S. J., Maund, J. R., Hendry, M. A., et al. 2004, Science, 303, 499

Smartt, S. J., Eldridge, J. J., Crockett, R. M., \& Maund, J. R. 2009, MNRAS, 395, 1409

Spiro, S., Pastorello, A., Pumo, M. L., et al. 2014, MNRAS, 439, 2873

Stanishev, V. 2007, Astron. Nachr., 328, 948

Tonry, J. L., Denneau, L., Heinze, A. N., et al. 2018, PASP, 130, 064505 van Dyk, S. D., Li, W., \& Filippenko, A. V. 2003, PASP, 115, 1289 van Dyk, S. D., Davidge, T. J., Elias-Rosa, N., et al. 2012, AJ, 143, 19 Vinkó, J., Sárneczky, K., Balog, Z., et al. 2009, ApJ, 695, 619 Yamanaka, M. 2018, Transient Name Server Classification Rep., 432 Zapartas, E. 2018, PhD Thesis, Univ. of Amsterdam, The Netherlands 
Table A.2. Photometric journal detailing the ATLAS observations of SN 2018aoq.

\begin{tabular}{|c|c|c|c|c|}
\hline Date & $\begin{array}{c}\text { MJD } \\
(+2400000)\end{array}$ & $\begin{array}{r}\text { Epoch } \\
\text { (d) }\end{array}$ & Filter & Magnitude \\
\hline 2018 Mar 11 & 58188.50 & -19.50 & $o$ & $>18.06$ \\
\hline 2018 Mar 16 & 58194.46 & -13.54 & $c$ & $>20.37$ \\
\hline 2018 Mar 19 & 58196.50 & -11.50 & $c$ & $>19.87$ \\
\hline 2018 Mar 28 & 58206.47 & -1.53 & $o$ & $>18.89$ \\
\hline 2018 Apr 05 & 58214.47 & 6.47 & $o$ & $15.30(0.01)$ \\
\hline 2018 Apr 11 & 58220.45 & 12.45 & $o$ & $15.34(0.01)$ \\
\hline 2018 Apr 14 & 58222.53 & 14.53 & $o$ & $15.33(0.01)$ \\
\hline 2018 Apr 15 & 58224.46 & 16.46 & $c$ & $15.56(0.02)$ \\
\hline 2018 Apr 23 & 58232.43 & 24.43 & $o$ & $15.41(0.01)$ \\
\hline 2018 Apr 25 & 58234.39 & 26.39 & $o$ & $>15.36$ \\
\hline 2018 Apr 25 & 58234.41 & 26.41 & $o$ & $15.33(0.32)$ \\
\hline 2018 May 05 & 58244.41 & 36.41 & $o$ & $15.46(0.02)$ \\
\hline 2018 May 07 & 58246.42 & 38.42 & $o$ & $15.35(0.01)$ \\
\hline 2018 May 09 & 58248.41 & 40.41 & $o$ & $15.40(0.01)$ \\
\hline 2018 May 11 & 58250.40 & 42.40 & $o$ & $15.33(0.01)$ \\
\hline 2018 May 13 & 58251.51 & 43.51 & $c$ & $15.68(0.01)$ \\
\hline 2018 May 13 & 58252.48 & 44.48 & $c$ & $15.67(0.01)$ \\
\hline 2018 May 15 & 58254.42 & 46.42 & $o$ & $15.40(0.01)$ \\
\hline 2018 May 17 & 58256.40 & 48.40 & $c$ & $15.66(0.01)$ \\
\hline 2018 May 19 & 58258.43 & 50.43 & $o$ & $15.35(0.01)$ \\
\hline 2018 May 21 & 58260.43 & 52.43 & $o$ & $15.42(0.06)$ \\
\hline 2018 May 23 & 58262.36 & 54.36 & $o$ & $15.53(0.27)$ \\
\hline 2018 May 25 & 58264.37 & 56.37 & $o$ & $15.36(0.02)$ \\
\hline 2018 May 27 & 58266.36 & 58.36 & $o$ & $15.34(0.05)$ \\
\hline 2018 May 31 & 58270.34 & 62.34 & $o$ & $15.37(0.04)$ \\
\hline 2018 Jun 02 & 58272.34 & 64.34 & $o$ & $15.35(0.01)$ \\
\hline 2018 Jun 04 & 58274.33 & 66.33 & $o$ & $15.32(0.01)$ \\
\hline 2018 Jun 06 & 58276.32 & 68.32 & $o$ & $15.32(0.02)$ \\
\hline 2018 Jun 08 & 58278.31 & 70.31 & $o$ & $15.32(0.01)$ \\
\hline 2018 Jun 10 & 58280.34 & 72.34 & $c$ & $16.36(0.02)$ \\
\hline 2018 Jun 12 & 58282.30 & 74.30 & $o$ & $15.36(0.01)$ \\
\hline 2018 Jun 14 & 58284.33 & 76.33 & $c$ & $16.39(0.02)$ \\
\hline 2018 Jun 16 & 58286.28 & 78.28 & $o$ & $15.36(0.01)$ \\
\hline 2018 Jun 18 & 58288.28 & 80.28 & $o$ & $15.41(0.01)$ \\
\hline 2018 Jun 20 & 58290.27 & 82.27 & $o$ & $15.53(0.01)$ \\
\hline 2018 Jun 22 & 58292.27 & 84.27 & $o$ & $15.52(0.02)$ \\
\hline 2018 Jun 24 & 58294.27 & 86.27 & $o$ & $15.56(0.02)$ \\
\hline 2018 Jun 26 & 58296.29 & 88.29 & $o$ & $15.55(0.01)$ \\
\hline 2018 Jun 27 & 58297.32 & 89.32 & $o$ & $15.62(0.04)$ \\
\hline 2018 Jun 28 & 58298.26 & 90.26 & $o$ & $15.63(0.02)$ \\
\hline 2018 Jun 30 & 58300.27 & 92.27 & $o$ & $15.69(0.01)$ \\
\hline 2018 Jul 02 & 58302.28 & 94.28 & $o$ & $15.67(0.01)$ \\
\hline 2018 Jul 04 & 58304.26 & 96.26 & $o$ & $15.70(0.05)$ \\
\hline 2018 Jul 10 & 58310.26 & 102.26 & $o$ & $15.79(0.06)$ \\
\hline 2018 Jul 12 & 58312.28 & 104.28 & $c$ & $18.05(0.13)$ \\
\hline 2018 Jul 16 & 58316.28 & 108.28 & $c$ & $>20.13$ \\
\hline 2018 Jul 18 & 58318.26 & 110.26 & $o$ & $16.14(0.04)$ \\
\hline 2018 Jul 20 & 58320.26 & 112.26 & $o$ & $16.96(0.19)$ \\
\hline 2018 Jul 22 & 58322.26 & 114.26 & $o$ & $16.67(0.05)$ \\
\hline 2018 Jul 24 & 58324.26 & 116.26 & $o$ & $17.29(0.07)$ \\
\hline 2018 Jul 30 & 58330.25 & 122.25 & $o$ & $17.66(0.13)$ \\
\hline 2018 Jul 31 & 58331.30 & 123.30 & $c$ & $>16.56$ \\
\hline 2018 Aug 07 & 58338.24 & 130.24 & $o$ & $17.98(0.38)$ \\
\hline 2018 Aug 13 & 58344.25 & 136.25 & $c$ & $>18.00$ \\
\hline
\end{tabular}

Notes. Measurements from images taken in the same filter and on the same night were averaged. 
Table A.3. Record of the archival WFC3 images used to identify the progenitor.

\begin{tabular}{ccccccc}
\hline \hline Date & $\begin{array}{c}\text { MJD } \\
(+2400000)\end{array}$ & $\begin{array}{c}\text { Phase } \\
(\mathrm{d})\end{array}$ & Instrument & Filter & $\begin{array}{c}\text { Exposure time } \\
(\mathrm{s})\end{array}$ & Vega mag. \\
\hline 2015 Dec 07 & 57363.06 & -845 & WFC3/UVIS & F350LP & 1050 & $25.67(0.06)$ \\
2015 Dec 07 & 57363.08 & -845 & WFC3/IR & F160W & 1106 & $21.79(0.02)$ \\
2015 Dec 14 & 57370.68 & -837 & WFC3/UVIS & F350LP & 1050 & $25.69(0.04)$ \\
2015 Dec 14 & 57370.70 & -837 & WFC3/UVIS & F555W & 1100 & $26.61(0.11)$ \\
2015 Dec 21 & 57377.92 & -830 & WFC3/UVIS & F814W & 1100 & $23.77(0.04)$ \\
2015 Dec 28 & 57384.74 & -823 & WFC3/IR & F160W & 1106 & $21.89(0.02)$ \\
2016 Jan 03 & 57390.02 & -818 & WFC3/UVIS & F350LP & 1050 & $25.83(0.05)$ \\
2016 Jan 03 & 57390.04 & -818 & WFC3/UVIS & F555W & 1100 & $26.77(0.12)$ \\
2016 Jan 05 & 57392.77 & -815 & WFC3/UVIS & F350LP & 1050 & $25.69(0.04)$ \\
2016 Jan 12 & 57399.14 & -809 & WFC3/UVIS & F814W & 1100 & $24.10(0.05)$ \\
2016 Feb 05 & 57423.56 & -784 & WFC3/UVIS & F350LP & 1050 & $25.81(0.04)$ \\
2016 Feb 11 & 57429.54 & -778 & WFC3/UVIS & F814W & 1100 & $24.07(0.04)$ \\
2016 Feb 18 & 57436.89 & -771 & WFC3/UVIS & F350LP & 1050 & $25.76(0.04)$ \\
2018 Apr 23 & 58232.17 & +24 & WFC3/UVIS & F555W & 270 & $15.79(0.01)$ \\
\hline
\end{tabular}

Notes. ${ }^{(a)}$ This image contains the SN (see proposal 15151).

Table A.4. Details of spectroscopic observations of SN 2018aoq.

\begin{tabular}{lcrc}
\hline \hline Date & $\begin{array}{c}\text { MJD } \\
(+2400000)\end{array}$ & $\begin{array}{r}\text { Phase } \\
(\mathrm{d})\end{array}$ & $\begin{array}{c}\text { Exposure Time } \\
(\mathrm{s})\end{array}$ \\
\hline 2018 Apr 03 & 58211.64 & 4 & 1200 \\
2018 Apr 05 & 58213.61 & 6 & 1200 \\
2018 Apr 17 & 58225.51 & 18 & 1200 \\
2018 May 11 & 58249.52 & 42 & 1200 \\
2018 May 30 & 58269.43 & 61 & 1200 \\
2018 Jul 19 & 58319.39 & 111 & 1800 \\
\hline
\end{tabular}

Notes. All spectra were taken using the Nordic Optical Telescope ALFOSC as part of the NUTS programme. Grism 4 (3200-9600Å) was used in a combination with a $1^{\prime \prime} .0$ slit providing a resolution of $16.2 \AA$. The second order contamination of grism 4 was corrected using the prescriptions of Stanishev (2007).

Table A.5. Photometry of the reference stars as labelled in Fig. A.1.

\begin{tabular}{cccccccccc}
\hline \hline No. & RA & Dec & $u$ & $B$ & $V$ & $g$ & $r$ & $i$ & $z$ \\
\hline 1 & $12: 10: 38.604$ & $+39: 20: 29.725$ & 16.17 & 15.60 & 14.46 & 15.02 & 14.08 & 12.19 & 12.61 \\
2 & $12: 10: 16.845$ & $+39: 19: 24.478$ & 17.98 & 16.67 & 15.80 & 16.20 & 15.54 & 15.32 & 15.22 \\
3 & $12: 10: 12.437$ & $+39: 19: 34.249$ & 14.83 & 13.73 & 12.83 & 13.25 & 12.56 & 12.43 & 13.08 \\
4 & $12: 10: 23.596$ & $+39: 21: 24.185$ & 16.90 & 15.81 & 15.08 & 15.40 & 14.88 & 14.72 & 14.63 \\
5 & $12: 10: 25.285$ & $+39: 23: 55.432$ & 16.17 & 15.11 & 14.39 & 14.70 & 14.19 & 14.07 & 14.02 \\
6 & $12: 10: 30.733$ & $+39: 25: 36.616$ & 15.51 & 14.17 & 14.58 & 14.21 & 14.85 & 16.14 & 13.55 \\
7 & $12: 10: 37.775$ & $+39: 25: 09.376$ & 20.50 & 18.51 & 17.21 & 17.87 & 16.76 & 16.35 & 16.12 \\
8 & $12: 10: 39.930$ & $+39: 23: 52.103$ & 18.76 & 18.18 & 17.68 & 17.86 & 17.58 & 17.47 & 17.44 \\
9 & $12: 10: 48.489$ & $+39: 26: 54.815$ & 22.84 & 20.82 & 19.14 & 20.03 & 18.54 & 17.21 & 16.51 \\
10 & $12: 10: 55.625$ & $+39: 26: 24.242$ & 18.34 & 17.50 & 16.87 & 17.13 & 16.71 & 16.54 & 16.47 \\
\hline
\end{tabular}


Table A.6. II-P sample used to calculate extinction.

\begin{tabular}{ccc}
\hline \hline SN & $E(B-V)$ & Reference \\
\hline $2005 \mathrm{cs}$ & 0.050 & Baron et al. (2007) \\
$1999 \mathrm{em}$ & 0.100 & Hamuy et al. (2001) \\
$1999 \mathrm{br}$ & 0.024 & NED, Pastorello et al. (2004) \\
$2009 \mathrm{md}$ & 0.100 & Fraser et al. (2011) \\
$2003 \mathrm{gd}$ & 0.100 & Hendry et al. (2005) \\
$2004 \mathrm{eg}$ & 0.399 & Spiro et al. (2014) \\
$2008 \mathrm{in}$ & 0.098 & Roy et al. (2011) \\
$2004 \mathrm{dj}$ & 0.100 & Vinkó et al. (2009) \\
\hline
\end{tabular}

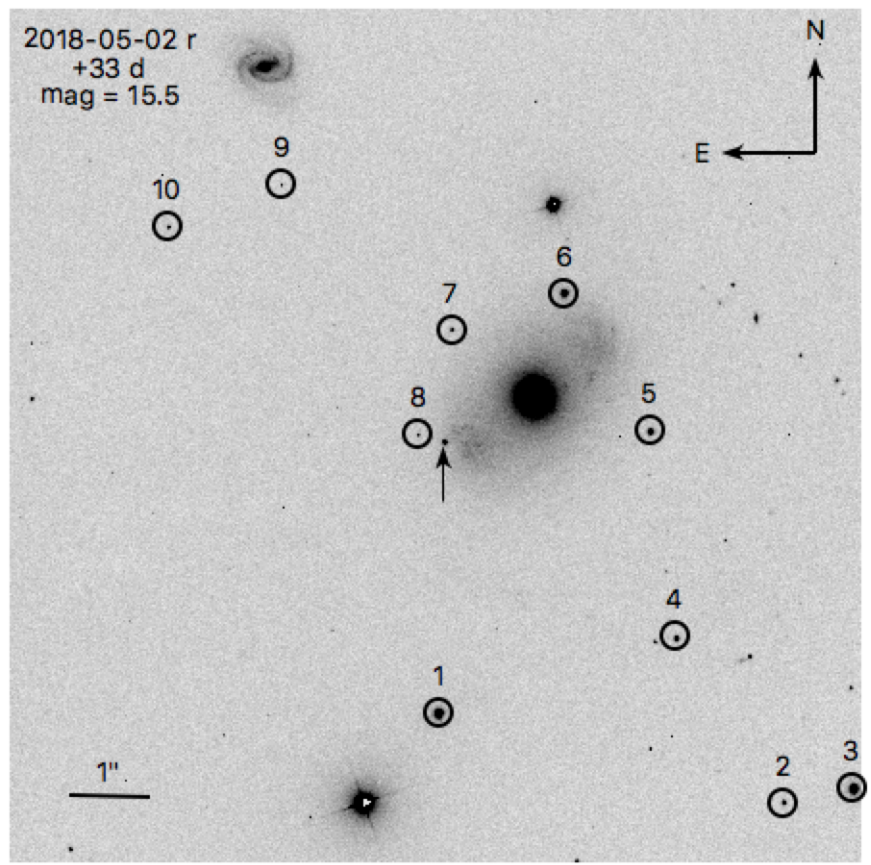

Fig. A.1. LT IO:O $r$-band image showing the location of SN 2018aoq indicated by the arrow. It is located at $\alpha_{J 2000}=12^{\mathrm{h}} 10^{\mathrm{m}} 38^{\mathrm{s}} .190, \delta_{J 2000}=$ $+39^{\circ} 23^{\prime} 47^{\prime \prime} 00$, or $65^{\prime \prime} .0 \mathrm{E}$ and $33^{\prime \prime} 6 \mathrm{~S}$ of the nucleus of NGC 4151 . The reference stars used for calibration are encircled and listed in Table A.5.

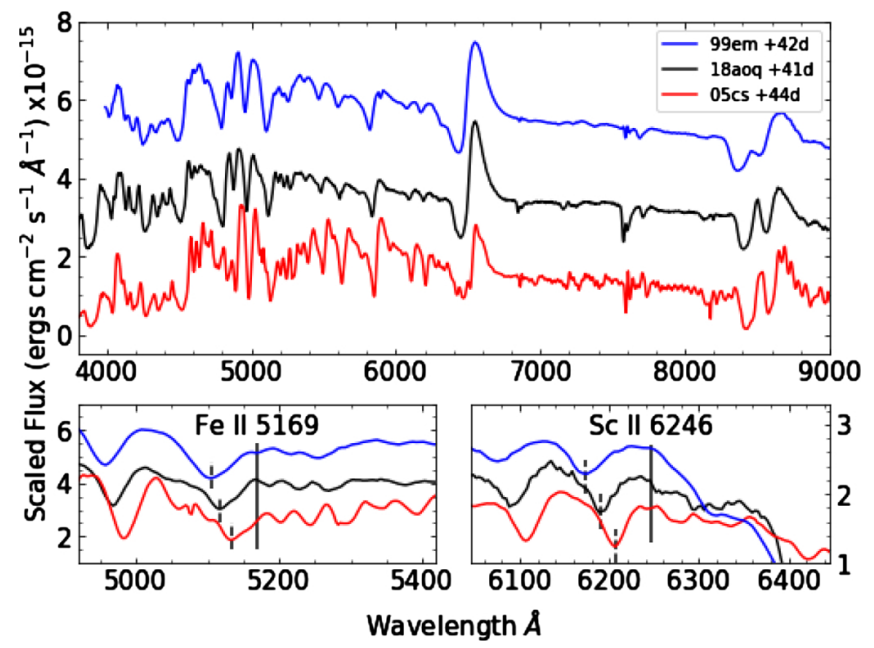

Fig. A.2. Spectral comparison of SN 2018aoq alongside a normal IIP (SN 1999em) and a low luminosity IIP (SN 2005cs). The bottom panels show the Fe II and $\mathrm{Sc}$ II absorption in more detail. Dashed lines indicate the minimum of the absorption feature. The solid line indicates the rest wavelength of the feature.

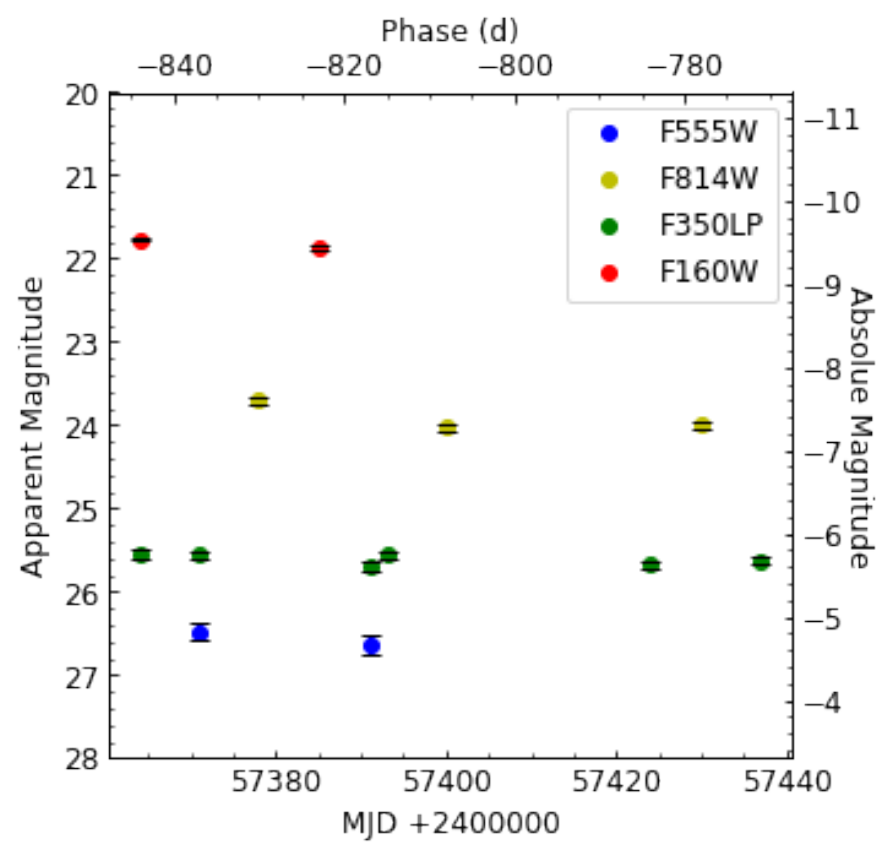

Fig. A.3. Extinction corrected light curves of the progenitor candidate.

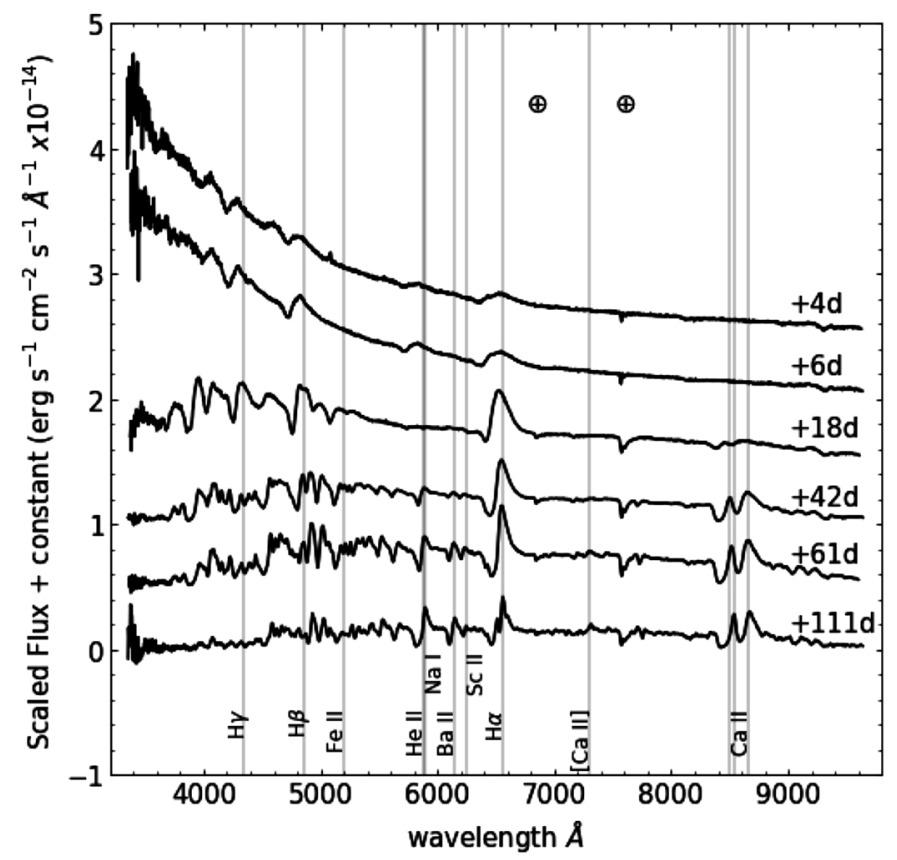

Fig. A.4. Spectral evolution of SN 2018aoq over 4 months. The spectra have been offset for clarity and corrected for both redshift and extinction. 